\title{
TITLE:
}

\section{Clinical and immunological characterization of paraneoplastic retinopathy.}

\section{AUTHOR(S):}

Makiyama, Yukiko; Kikuchi, Takanobu; Otani, Atsushi; Oishi, Akio; Guo, Congrong; Nakagawa, Satoko; Ogino, Ken; Kojima, Hiroshi; Kurimoto, Masafumi; Yoshimura, Nagahisa

\section{CITATION:}

Makiyama, Yukiko ...[et al]. Clinical and immunological characterization of paraneoplastic retinopathy.. Investigative ophthalmology \& visual science 2013, 54(8): 5424-5431

\section{ISSUE DATE:}

2013-08

URL:

http://hdl.handle.net/2433/187393

\section{RIGHT:}

(c) Association for Research in Vision and Ophthalmology; この論文は出 版社版でありません。引用の際には出版社版をご確認ご利用ください 。; This is not the published version. Please cite only the published version. 


\section{Clinical and Immunological Characterization of Paraneoplastic Retinopathy}

Short title: Characterization of Paraneoplastic Retinopathy

Yukiko Makiyama, ${ }^{1}$ Takanobu Kikuchi, ${ }^{2}$ Atsushi Otani, ${ }^{1}$ Akio Oishi, ${ }^{1}$ Congrong Guo, ${ }^{1}$ Satoko Nakagawa, ${ }^{1}$ Ken Ogino, ${ }^{1}$ Hiroshi Kojima, ${ }^{1}$ Masafumi Kurimoto, ${ }^{1}$ Nagahisa Yoshimura, ${ }^{1}$

${ }^{1}$ Department of Ophthalmology, Kyoto University Graduate School of Medicine, Kyoto 606-8507, Japan

${ }^{2}$ Department of Instrumental Analysis Research Center for Human and Environmental Science, Shinshu Univeisity, Nagano 390-8621, Japan

All correspondence should be addressed to:

Atsushi Otani, MD, PhD

Department of Ophthalmology, Kyoto University Graduate School of Medicine

54-Kawaharacho, Shyogoin, Sakyo-ku, Kyoto 606-8507, Japan

E-mail: otan@kuhp.kyoto-u.ac.jp, Fax:+81-75-752-0933

Tel: $+81-75-751-3248$ 
Key words: paraneoplastic retinopathy, spectral domain-optical coherence tomography, Western blot analysis, immunohistochemistory, autoantibody, anti-retinal antibody 


\section{Abstract}

Purpose: To report the clinical and immunological characterization of paraneoplastic retinopathy and to investigate the association between spectral domain-optical coherence tomography (SD-OCT) findings and the target of autoantibodies in paraneoplastic retinopathy (PR).

Design: Observational case series.

Methods: We retrospectively enrolled 8 patients (age, 57-85 years; 4 men and 4 women) suspected to have PR. All patients underwent comprehensive ophthalmic examinations, including best-corrected visual acuity (BCVA) measurement, slit-lamp examinations, kinetic visual field testing with Goldmann perimetry (GP), electroretinography (ERG), fundus photography, fluorescein angiography (FA), fundus autofluorescence (FAF), SD-OCT, and experimental serum examinations (Western blot analysis and immunohistochemistry [IHC]).

Results: Three patients had previous history of malignant tumors, and 4 were newly diagnosed with neoplastic tumors (small cell lung carcinoma, thymoma, pancreatic neuroendocrine neoplasm, and colon cancer). Another de novo malignancy (small cell lung carcinoma) was detected in 1 patient with a previous history of malignancy (bladder cancer and liposarcoma). BCVA in these patients ranged from hand motion to 1.5. The GP revealed island, ring-shaped, concentric, or central scotoma. All the patients showed non-recordable or reduced amplitude ERG. Fluorescein leakage was detected in 5 patients. Hyperand/or hypo- autofluorescence on FAF was detected in 6 cases. The serum examinations identified anti-retinal antibodies in all of the patients. Patients whose serum contained anti-photoreceptor or anti-RPE antibody as evidenced with IHC showed damages of outer retina in OCT.

Conclusions: In this case series, PR was associated with a variety of neoplasms and autoantibodies. SD-OCT can be used to characterize morphologic changes and the changes were associated with the target of autoantibodies. 


\section{Introduction}

Autoimmune retinopathy (AIR) is a progressive retinal degeneration caused by autoimmune processes. These processes are considered to be mediated by autoantibodies directed to retinal proteins. AIR cases with underlying malignant or benign tumors are termed paraneoplastic retinopathies (PRs). This classification includes cancer-associated retinopathy (CAR), melanoma-associated retinopathy $(\mathrm{MAR}),{ }^{1}$ and lymphoma-associated retinopathy $(\mathrm{LAR}) .^{2}$ One mechanism for $\mathrm{PR}$ development is hypothesized as the production of autoantibodies against common proteins expressed in both the neoplasm and the retina. ${ }^{3} 4$

PR was first described in 1976 by Sawyer et al. ${ }^{5}$ Subsequent case reports classified characteristic clinical features including a sudden and progressive loss of vision associated with photopsia, ring scotoma or concentric visual field defects, attenuated retinal arterioles, and abnormal electroretinograms (ERG). ${ }^{1}$ The presence of circulating serum autoantibodies specific to retinal antigens is necessary for a diagnosis of PR; however, this laboratory test is not available in most ophthalmological settings. ${ }^{6}$ Western blot can be used to detect anti-retinal antibodies. ${ }^{7}$ However, the lack of standardization across studies makes it difficult to compare the results obtained by various authors. ${ }^{8-10}$

Recent progress in imaging technology has allowed us to detect detailed retinal changes and provided insight into the pathological mechanisms underlying retinal diseases. Optical coherence tomography (OCT), which allows for the noninvasive visualization of normal retina as well as of pathologic conditions, is now widely accepted as a standard tool in clinical practice. ${ }^{11}$ The currently available spectral domain-OCT (SD-OCT) machines achieve resolution up to $5 \mu \mathrm{m}$; indeed, recent SD-OCT devices allow for "layer by layer" evaluation of the retina. ${ }^{12,13}$ Previous reports showed that various imaging modalities can be used to strengthen a diagnosis of CAR or PR. ${ }^{14,15}$ However, there is limited information about the SD-OCT findings associated with PRs. One of the purposes of this study is to find any clinical characteristic features including SD-OCT findings seen in patients with PR. We also investigated the association with clinical characteristics and serum testing (e.g., Western blot analysis and immunohistochemistry [ $\mathrm{IHC}]$ ) to confirm the diagnosis and to understand the molecular basis of the pathologic changes. 


\section{Methods}

\section{Subjects and clinical examinations}

The study comprised of 8 patients ( 4 men and 4 women) who were seen at Kyoto University Hospital (Kyoto, Japan) from June 2008 to August 2011 and who were suspected to have PR. The study was conducted in accordance with the tenets of the Declaration of Helsinki. The study design was approved by the Ethics committee of Kyoto University. Two of the 8 patients were presented in a prior case report without describing Western blot data and OCT findings. ${ }^{16}$

PR was suspected when a patient showed rapidly progressing vision loss and/or a visual acuity defect, which cannot be explained by fundus examination findings or the pathophysiology of other retinal or optic nerve diseases. The attenuation of retinal arterioles or the presence of photophobia was considered to support a diagnosis of PR. In such cases, we performed Goldmann perimetry (GP), ERG, fluorescein angiography (FA), fundus autofluorescence imaging (FAF), and SD-OCT. Systemic screening was accomplished using computed tomography (CT). Additional examinations with endoscopy or bronchoscopy were performed as needed. Informed consent was obtained from the participants after the objectives and the nature of the procedures had been explained fully; blood samples were collected thereafter. We diagnosed patients as having PR when they showed: (1) reduced amplitude of ERG; (2) presence of anti-retinal autoantibodies; (3) previous or newly detected neoplasm; (4) acute or subacute disease progression.

Retinal section images were acquired using a Spectralis $\mathrm{HRA}_{+} \mathrm{OCT}^{\circledR}$ (Heidelberg Engineering, Heidelberg, Germany). Built-in Automatic Real Time (ART) software was used to average images in order to improve the signal to noise ratio. We obtained $9-\mathrm{mm}$ cross sections setting the ART function to 100 frames per scan. We also acquired 9-mm radial sections centered on the fovea, for which 20 images were averaged. FAF and FA images were obtained using confocal angiography (HRA2; Heidelberg Engineering). Full-field ERGs were recorded using LS-C (Mayo Co, Nagoya, Japan) and Neuropack MEB-2204 (Nihon Kohden, Tokyo, Japan) software according to the ISCEV standard protocol recommended in $2008 .^{17}$

\section{Western Blot Analysis}

Serum was obtained from each patient and analyzed by Western blot as previously reported. ${ }^{18}$ Briefly, retina, brain, kidney, liver, and spleen tissue 
samples from C57BL/6 mice were homogenized. Extracted proteins were used to detect organ-specific antibodies. Proteins from each organ were separated by $8 \%$ or $10 \%$ SDS-PAGE electrophoresis and transferred to polyvinylidene difluoride (PVDF) membranes. The membrane was probed with the patient's serum, then rinsed and incubated with alkaline phosphatase-conjugated goat anti-human IgG (Jackson Immuno Research, West Grove, PA). The associated signal was detected with a BCIP (5-bromo-4-chloro-3'-indolylphosphatase p-toluidine salt) /NBT (nitro-blue tetrazolium chloride) (BCIP/NBT) detection system. When bands were observed in retina and/or brain lanes but not in the other lanes, the serum was judged as having anti-retina specific antibodies. In addition, the presence of anti-recoverin antibody was investigated using a commercial product provided by Athena Diagnostics (Worcester, MA).

\section{Immunohistochemistry (IHC)}

C57BL/6 mouse eyes were fixed by immersion at $4^{\circ} \mathrm{C}$ for $12 \mathrm{~h}$ in a freshly prepared solution of $0.5 \%$ zinc chloride, $17.16 \mathrm{mM}$ zinc trifluoroacetate, and $0.05 \%$ calcium acetate in $0.1 \mathrm{M}$ Tris- $\mathrm{HCl} \mathrm{pH} 6.5$, then embedded in paraffin. Four- $\mu$ m-thick sections were prepared, deparaffinized, and blocked with $4 \%$ normal goat serum, $0.1 \%$ Triton X-100, and 0.05\% NP-40 in PBS for $1 \mathrm{~h}$ at room temperature. The slides were then incubated with a 1:50 dilution of the patient's serum overnight at $4^{\circ} \mathrm{C}$. Next, the specimens were rinsed in PBS and incubated with the Alexa Fluor 488-conjugated anti-human IgG antibody (Invitrogen, Carlsbad, CA). In some cases, the retinal slices were co-incubated with rhodamine-conjugated peanut agglutinin (Sigma-Aldrich, St. Louis, MO), rabbit polyclonal antibodies against blue opsin (Chemicon, Temecula, CA) or S-100 protein (Dako, Glostrup, Denmark). These antibodies were used to label the outer segments of cone cells, the outer segments of s-cone cells and Müller cells, respectively. In these cases, Alexa Fluor 594-conjugated anti-rabbit IgG was used as a secondary antibody. The slides were finally mounted in a mounting medium containing diamidino-2-phenylindole (DAPI) (Vector Laboratories, Burlingame, CA). The contribution of secondary antibodies to the observed immune reaction was verified by staining without the primary antibody. The sections were examined using a confocal scanning microscope (LSM 5 Exciter, Carl Zeiss Microimaging, Jena, Germany).

\section{Genetic analysis}


Genomic DNAs were prepared from peripheral blood using a DNA extraction kit (QuickGene-610L; Fujifilm, Minato, Tokyo, Japan) according to the manufacturer's protocol, and the A260/A280 optical density was evaluated. The DNA from each patient except that of case 3 was submitted to genetic analysis to exclude the presence of genes linked to retinal degeneration: $C E R K L$, CNGA1, CNGB1, MERTK, PED6A, PED6B, PNR, RDH12, RGR, RLBP1, SAG, TULP1, CRB, RPE65, USH2A, USH3A, LRAT, PROML1, and PBP3. The tests were performed with using a microarray (Asper Opthalmics, Tartu, Estonia).

\section{Results}

\section{Clinical findings}

The clinical findings are summarized in Table. Initial BCVA ranged from hand motion to 1.5. All patients complained of reduced visual acuity or visual field defects. GP showed island, ring-shaped, concentric, or central scotoma.

The ERG results are shown in Figure 1. ERG revealed abnormalities in all cases, but the degree of impairment ranged from non-recordable to slight changes in the cone response in one eye.

Vasculitis as confirmed by fluorescein leakage from the vasculature was observed in 5 cases (cases 2, 3, 4, 5, and 7). Two patients (cases 3 and 4) also had vitreous opacities. FA revealed a petaloid leakage in case 4.

Three patients had previous history of malignant tumors (Bladder cancer, liposarcoma, thymoma, renal cancer, lymphoma, and renal cancer), and 4 were newly diagnosed with neoplastic tumors (small cell lung carcinoma, thymoma, pancreatic neuroendocrine neoplasm, and colon cancer). Another de novo malignancy (small cell lung carcinoma) was detected in 1 patient with a previous history of malignancy (bladder cancer and liposarcoma).

\section{Fundus autofluorescence findings}

In most cases, abnormality of FAF was detected. Abnormal hyper- and/or hypoautofluorescence was noted at presentation except for case 5 and 8 (Figure 2). Although cases 5 and 8 displayed no detectable abnormality at initial examination, case 8 developed abnormal hyper- and hypo- autofluorescent lesions in 3 years. Eventually, abnormal FAF was confirmed in 7 patients of 8 patients (87.5\%).

\section{OCT findings}


OCT images revealed evidence of retinal degeneration in all cases, especially in outer retina (Figure 2, right). The detected changes include the obscuration, the interruption, or the disappearance of the inner and outer segments junction of the photoreceptors (IS/OS). External limiting membrane (ELM) was also affected in case $1,2,3,4,5,7$, and 8 . Thinning of foveal or parafoveal outer nuclear layer was noted in case $2,3,4,6,7$, and 8 . Case 4 showed cystoid macular edema.

\section{Western blot}

The results of Western blot are shown in Figure 3 and Table. Western blot analysis detected anti-retinal antibodies in all sera samples. Several bands specific to retinal proteins were observed. Anti-recoverin antibody was present in case 2 . The molecular weights and the number of bands detected varied among patients.

\section{Comparison between Immunohistochemistry and SD-OCT}

High-magnification IHC and OCT images are shown in Figure 4. Abnormalities of outer retina were more easily confirmed in magnified SD-OCT images (Figure 4, upper panels). Patients' serum reacted to retinal tissue as shown in the lower panels of Figure 4.

In cases 2, 3, 4, and 5, SD-OCT showed disruption of outer retinal structures (Figure 4-2, 3, 4, and 5). IHC staining was detected in inner or outer segment in these cases (Figure 4-B, C, D, and E). In cases 7 and 8, IHC staining was detected in RPE layer (Figure 4-G, H) and OCT showed the obscuration of IS/OS line (Figure 4-7, 8). Case 1, which showed strong staining in the inner retinal layers on $\mathrm{IHC}$ had relatively preserved outer retina on SD-OCT image (Figure 4-1, A). In case 6, IHC staining was most prominent in the ganglion cell layer (Figure 4-F). SD-OCT image showed the thinning of outer nuclear layer, interruption of IS/OS (Figure 4-6). In addition, thinning of retinal nerve fiber layer was noted in this case (Figure 2-R).

\section{Genetic analysis}

To rule out genetic retinal degenerations, mutation screening was carried out by using microarray of Asper Opthalmics. Candidate gene mutations included CERKL, CNGA1, CNGB1, MERTK, PED6A, PED6B, PNR, RDH12, RGR, RLBP1, SAG, TULP1, CRB, RPE65, USH2A, USH3A, LRAT, PROML1, and $P B P 3$. No mutations were detected on these 7 patients by the analysis. Mutation 
screening was not perfomed in case 3 because informed consent about genetic analysis was not obtained.

\section{Discussion}

In the present study, we reported 8 patients with PR induced by various tumors. The underlying neoplasms that induced PR were bladder cancer, liposarcoma, thymoma, lymphoma, and renal cancer. In 5 patients, diagnoses of SCLC, thymoma, pancreatic neuroendocrine neoplasm, and colon cancer were made after patients presented with retinopathy. Although SCLC is the most common cause of PR, ${ }^{1,4,5,6,16,19-21}$ other systemic neoplasms including bladder cancer, thymoma, liposarcoma, and renal cancer have also been associated with PR. ${ }^{4,22}$ Neuroendocrine carcinoma of the fallopian tube has also been reported as a cause of PR. ${ }^{23}$ To our best knowledge, pancreatic neuroendocrine neoplasm has not been reported as a cause of PR.

In PR, autoantibodies against retinal antigens are considered to cause retinal dysfunction and retinal cell death. Anti-recoverin antibody, which recognizes a $23 \mathrm{kDa}$ retinal protein found in rod and cone photoreceptors as well as tumor cells, is the most studied autoantibody. ${ }^{20-22,24-27}$ Anti-recoverin autoantibodies bind to photoreceptor cells and triggers apoptosis. ${ }^{21,28,29}$ In the present study, anti-recoverin antibody was detected in only one patient (Case 2) and the other patients showed unidentified antibodies. The protein targets of autoantibodies reported to cause PR to date include retinal alpha-enolase (46 kDa); ${ }^{30-32}$ arrestin (48 kDa) ;3, 34 Tubby-like protein 1 (TULP1) (78 kDa); ${ }^{35}$ heat shock cognate protein (hsc) $70(65 \mathrm{kDa}){ }^{36,37}$ transducin beta $(35 \mathrm{kDa}){ }^{38}$ anti-carbonic anhydrase II (30 kDa); photoreceptor cell-specific nuclear receptor(46.5 kDa); ${ }^{39}$ interphotoreceptor retinoid binding protein $(145 \mathrm{kDa}){ }^{40}$ TRPM1 ( $\sim 180 \mathrm{kDa}$ or $\sim 200 \mathrm{kDa})^{41,42}$. A number of retinal antigen that were not identified have been described by western blot analysis. ${ }^{7,8,43}$ In the present study, anti-recoverin antibody was detected in only one patients and western blot detected a various bands. We were not able to determine antigens for various molecular weight proteins. However, for example, in case1, a band of molecular weight $36 \mathrm{kDa}$ was detected in Western blot, which was close to molecular weights of $35 \mathrm{kDa}$ reported by Peek et al as antigen against Müller cells ${ }^{44}$ and in fact, Müller cells were stained in this case. In case 5, Western blot detected a band of molecular weight $46 \mathrm{kDa}$. Although the protein was not identified, anti-alpha-enolase antibodies (46 kDa) were reported as a cause of 
cone dysfunction. ${ }^{32}$ Central visual field defect and almost non-recordable cone ERG in this patient is consistent with the report. Although it is not clear that all the detected bands have pathologic significance, the existence of these various autoantibodies, suggests that the pathology, symptoms, and clinical signs of PR are multi-faceted.

In the present study, SD-OCT images were compared to the results of IHC or Western blot. The patients with presumed anti-photoreceptor antibodies (cases 2, 3, 4, and 5) and anti-RPE antibodies (cases 7 and 8) exhibited severe damage to the outer retina and poor visual acuity. However, the patients without presumed anti-photoreceptor or anti-RPE antibodies (cases 1 and 6) exhibited relatively preserved IS/OS lines and had good visual acuity. This result is consistent with the report by Kondo et al. In their report, a patient with anti-TRPM1 antibody, which is expressed in ON bipolar cells, presented signals of INL and OPL but not photoreceptor degeneration in IHC and almost normal retinal structure in OCT images. ${ }^{42}$ The severe degeneration of photoreceptors depicted with SD-OCT might be associated with the presence of anti-photoreceptor or anti-RPE antibodies. Although this was an observational study and further investigations are needed, SD-OCT may facilitate the management of PR cases. ${ }^{15}$

FAF was also useful to detect the abnormality in patients with $P R$, as shown in previous reports. ${ }^{15,45}$ In fact, abnormal FAF was noted in 7 out of 8 patients. Variety of the appearance including perivascular hyperautofluorescence and mottled hypoautofluorescence or ring-shaped hyperautofluorescence was consistent with the previous report. ${ }^{15}$ In the present study, there was one patient who showed normal FAF. A patient with normal FAF was also noted in the previous report and the authors suggested several explanations, 1: the patient was identified very early in the disease process. 2 : the autoantibodies alter normal retinal function but did not cause cell loss. 3 : vision loss was due to inner retinal dysfunction rather than photoreceptor disruption or loss. ${ }^{15}$ In the present study, the first explanation above was not likely because abnormality of FAF was not observed even after 12 months. The second and third explanation was not applicable either, since OCT and ERG showed outer retinal abnormality. Further studies are required to understand the relationship between retinal dysfunction, pathologic process, and FAF finding in patients with PR.

It was reported that retinopathy sometimes precedes the detection of 
malignancy. ${ }^{4}$ In fact, neoplasms were detected after the presentation of retinopathy in 5 out of 8 patients in the present series. Thus, it is important to diagnose PR in a timely fashion. PR presented in our study shared the characteristics with previously reported cases: rapid, progressive, painless vision loss associated with photosensitivity that continued over a period ranging from weeks to months. In addition, patients with PR sometimes show asymmetric involvement in the right and left eyes, as shown in case 6 . This characteristic is atypical among patients with inherited retinal degeneration. Although it is still a challenge to diagnose PR in many cases because of the variety of clinical appearances and the technical difficulty of detecting autoantibodies in serum, ${ }^{8}$ clinicians should be careful when faced with a case with rapid, atypical or asymmetric retinal degeneration.

In addition to the difficulty of achieving an accurate PR diagnosis and despite the common use of steroids to treat PR, no definitive therapeutic protocol has been established. ${ }^{7}$ The prognosis is not good in many cases. In fact, 5 of 8 patients eventually display visual acuity worse than 0.1 in both eyes. Further investigations are warranted to establish effective treatment for this disease.

Some cases with CAR were reported to show specific ERG patterns. For example, negative ERG results were reported in patients with MAR or with anti-TRPM1 ( $\sim 180 \mathrm{kDa}$ or $\sim 200 \mathrm{kDa}$ ) antibody. ${ }^{42}$ In the current study, scotopic and photopic ERG results were equally affected in 7 cases, none of which exhibited a negative pattern. Adamus et al. reported that anti-enolase (46 kDa) antibody predominantly affected the photopic response, while anti-transducin (40 kDa) antibody predominantly affected the scotopic response, and anti-recoverin antibody reduced both responses. ${ }^{1}$ Anti-46 kDa protein antibody was seen in cases 5 and 7, scotopic and photopic ERG results were equally affected. Although case 6 exhibited a reduced cone response in the right eye, he did not have anti-46 kDa protein antibody. We did not find any specific correlation between laboratory data and ERG findings in this series of patients.

There are several limitations in this study. The first is its retrospective design and the small number of patients. The second limitation is that we were not able to identify the anti-retinal antibody. Considering that Shimazaki et al. reported the presence of anti-retinal antibodies in normal sera, ${ }^{46}$ all the antibodies detected in the present report might not always be the cause of the patient's PR. With regard to the anti-retinal antibody, there is another limitation; 
Western blot and IHC were performed using mice retina. Although the human retina would be most suitable for the detection of autoantibodies, human sample was not available. Considering that a previous study indicates that the retina from other species can be a substitute as long as there is an appropriate control, ${ }^{8}$ we assume that the effect of using mice retina instead of human retina should be minimal. In fact, most previous studies used bovine, pig, monkey, rat, and mouse as well as human retina. ${ }^{7,18,20,31,35,36,40,44,47,48}$ The third limitation is the clinical diagnosis of PR. Although we screened major genetic mutations and the presentation is not typical, we could not completely rule out genetic retinal degenerations in our cases.

Finally, we reported the result of clinical findings and experimental serum examinations in 8 patients with PR. Although there was a variety in morphologic changes depicted with SD-OCT as well as in targets of autoantibodies, SD-OCT and IHC findings showed some correlation. SD-OCT might be a potential tool for further investigation of the pathophysiology associated with PR. 
References

1. Adamus $\mathrm{G}$, Ren $\mathrm{G}$, Weleber RG. Autoantibodies against retinal proteins in paraneoplastic and autoimmune retinopathy. BMC Ophthalmol 2004;4:5.

2. Matus G, Dicato M, Focan C. [Cancer associated retinopathy (CAR). Two clinical cases and review of the literature]. Rev Med Liege 2007;62:166-169.

3. Adamus G. Autoantibody-induced apoptosis as a possible mechanism of autoimmune retinopathy. Autoimmun Rev 2003;2:63-68.

4. Adamus G. Autoantibody targets and their cancer relationship in the pathogenicity of paraneoplastic retinopathy. Autoimmun Rev 2009;8:410-414.

5. Sawyer RA, Selhorst JB, Zimmerman LE, Hoyt WF. Blindness caused by photoreceptor degeneration as a remote effect of cancer. Am J Ophthalmol 1976;81:606-613.

6. Jacobson DM, Thirkill CE, Tipping SJ. A clinical triad to diagnose paraneoplastic retinopathy. Ann Neurol 1990;28:162-167.

7. Ferreyra HA, Jayasundera T, Khan NW, He S, Lu Y, Heckenlively JR. Management of autoimmune retinopathies with immunosuppression. Arch Ophthalmol 2009;127:390-397.

8. Forooghian F, Macdonald IM, Heckenlively JR, et al. The need for standardization of antiretinal antibody detection and measurement. $A m \mathrm{~J}$ Ophthalmol 2008;146:489-495.

9. Jampol LM, Fishman GA. Immunosuppression for autoimmune retinopathy. Arch Ophthalmol 2009;127:573-575.

10. Heckenlively JR, Ferreyra HA, Jayasundera $T$, Janasundera $T$. Controversies of diagnosing autoimmune retinopathy. Arch Ophthalmol 2010;128:147-148; author reply 148-149.

11. Coker JG, Duker JS. Macular disease and optical coherence tomography. Curr Opin Ophthalmol 1996;7:33-38.

12. Drexler $W$, Sattmann $H$, Hermann $B$, et al. Enhanced visualization of macular pathology with the use of ultrahigh-resolution optical coherence tomography. Arch Ophthalmol 2003;121:695-706.

13. Ko TH, Fujimoto JG, Schuman JS, et al. Comparison of ultrahigh- and standard-resolution optical coherence tomography for imaging macular pathology. Ophthalmology 2005;112:1922.e1921-1915.

14. Shildkrot Y, Sobrin L, Gragoudas ES. Cancer-associated retinopathy: 
update on pathogenesis and therapy. Semin Ophthalmol 2011;26:321-328.

15. Pepple KL, Cusick M, Jaffe GJ, Mruthyunjaya P. SD-OCT and autofluorescence characteristics of autoimmune retinopathy. $\mathrm{Br} J$ Ophthalmol 2013;97:139-144.

16. Sakamori Y, Kim YH, Okuda C, et al. Two cases of cancer-associated retinopathy combined with small-cell lung cancer. Jpn $J$ Clin Oncol 2011;41:669-673.

17. Marmor MF, Fulton AB, Holder GE, et al. ISCEV Standard for full-field clinical electroretinography (2008 update). Doc Ophthalmol 2009;118:69-77.

18. Ohta K, Kikuchi T, Yoshida N. Slowly progressive non-neoplastic autoimmune-like retinopathy. Graefes Arch Clin Exp Ophthalmol 2011;249:155-158.

19. Matsuo S, Ohguro H, Ohguro I, Nakazawa M. Clinicopathological Roles of Aberrantly Expressed Recoverin in Malignant Tumor Cells. Ophthalmic Research 2010;43.

20. Thirkill CE, Tait RC, Tyler NK, Roth AM, Keltner JL. The cancer-associated retinopathy antigen is a recoverin-like protein. Invest Ophthalmol Vis Sci 1992;33:2768-2772.

21. Adamus G, Guy J, Schmied JL, Arendt A, Hargrave PA. Role of anti-recoverin autoantibodies in cancer-associated retinopathy. Invest Ophthalmol Vis Sci 1993;34:2626-2633.

22. Matsuo S, Ohguro H, Ohguro I, Nakazawa M. Clinicopathological roles of aberrantly expressed recoverin in malignant tumor cells. Ophthalmic Res 2010;43:139-144.

23. Raghunath A, Adamus G, Bodurka DC, Liu J, Schiffman JS. Cancer-associated retinopathy in neuroendocrine carcinoma of the fallopian tube. J Neuroophthalmol 2010;30:252-254.

24. Keltner JL, Thirkill CE. Cancer-associated retinopathy vs recoverin-associated retinopathy. Am J Ophthalmol 1998;126:296-302.

25. Heckenlively JR, Fawzi AA, Oversier J, Jordan BL, Aptsiauri N. Autoimmune retinopathy: patients with antirecoverin immunoreactivity and panretinal degeneration. Arch Ophthalmol 2000;118:1525-1533.

26. Bazhin AV, Schadendorf D, Philippov PP, Eichmüller SB. Recoverin as a cancer-retina antigen. Cancer Immunol Immunother 2007;56:110-116.

27. Thirkill CE, Tait RC, Tyler NK, Roth AM, Keltner JL. Intraperitoneal cultivation of small-cell carcinoma induces expression of the retinal 
cancer-associated retinopathy antigen. Arch Ophthalmol 1993;111:974-978.

28. Adamus G, Machnicki M, Seigel GM. Apoptotic retinal cell death induced by antirecoverin autoantibodies of cancer-associated retinopathy. Invest Ophthalmol Vis Sci 1997;38:283-291.

29. Chen W, Elias RV, Cao W, Lerious V, McGinnis JF. Anti-recoverin antibodies cause the apoptotic death of mammalian photoreceptor cells in vitro. J Neurosci Res 1999;57:706-718.

30. Adamus G, Aptsiauri N, Guy J, Heckenlively J, Flannery J, Hargrave PA. The occurrence of serum autoantibodies against enolase in cancer-associated retinopathy. Clin Immunol Immunopathol 1996;78:120-129.

31. Ren G, Adamus G. Cellular targets of anti-alpha-enolase autoantibodies of patients with autoimmune retinopathy. J Autoimmun 2004;23:161-167.

32. Weleber RG, Watzke RC, Shults WT, et al. Clinical and electrophysiologic characterization of paraneoplastic and autoimmune retinopathies associated with antienolase antibodies. Am $J$ Ophthalmol 2005;139:780-794.

33. Gilmour MA, Cardenas MR, Blaik MA, Bahr RJ, McGinnis JF. Evaluation of a comparative pathogenesis between cancer-associated retinopathy in humans and sudden acquired retinal degeneration syndrome in dogs via diagnostic imaging and western blot analysis. Am J Vet Res 2006;67:877-881.

34. Heckenlively JR, Jordan BL, Aptsiauri N. Association of antiretinal antibodies and cystoid macular edema in patients with retinitis pigmentosa. $A m \mathrm{~J}$ Ophthalmol 1999;127:565-573.

35. Kikuchi T, Arai J, Shibuki H, Kawashima H, Yoshimura N. Tubby-like protein 1 as an autoantigen in cancer-associated retinopathy. J Neuroimmunol 2000;103:26-33.

36. Ohguro H, Ogawa K, Maeda T, Maeda A, Maruyama I. Cancer-associated retinopathy induced by both anti-recoverin and anti-hsc70 antibodies in vivo. Invest Ophthalmol Vis Sci 1999;40:3160-3167.

37. Ohguro H, Ogawa K, Nakagawa T. Recoverin and Hsc 70 are found as autoantigens in patients with cancer-associated retinopathy. Invest Ophthalmol Vis Sci 1999;40:82-89.

38. Potter MJ, Adamus G, Szabo SM, Lee R, Mohaseb K, Behn D. Autoantibodies to transducin in a patient with melanoma-associated retinopathy. Am J Ophthalmol 2002;134:128-130.

39. Eichen JG, Dalmau J, Demopoulos A, Wade D, Posner JB, Rosenfeld 
MR. The photoreceptor cell-specific nuclear receptor is an autoantigen of paraneoplastic retinopathy. J Neuroophthalmol 2001;21:168-172.

40. Querques G, Thirkill CE, Hagege H, Soubrane G, Souied EH. Choroidal neovascularization associated with cancer-associated retinopathy. Acta Ophthalmol 2010;88:571-575.

41. Dhingra A, Fina ME, Neinstein $A$, et al. Autoantibodies in melanoma-associated retinopathy target TRPM1 cation channels of retinal ON bipolar cells. J Neurosci 2011;31:3962-3967.

42. Kondo M, Sanuki $R$, Ueno $S$, et al. Identification of autoantibodies against TRPM1 in patients with paraneoplastic retinopathy associated with ON bipolar cell dysfunction. PLoS One 2011;6:e19911.

43. Thirkill CE, Roth AM, Keltner JL. Cancer-associated retinopathy. Arch Ophthalmol 1987;105:372-375.

44. Peek R, Verbraak F, Coevoet HM, Kijlstra A. Müller cell-specific autoantibodies in a patient with progressive loss of vision. Invest Ophthalmol Vis Sci 1998;39:1976-1979.

45. Lima LH, Greenberg JP, Greenstein VC, et al. Hyperautofluorescent ring in autoimmune retinopathy. Retina 2012;32:1385-1394.

46. Shimazaki K, Jirawuthiworavong GV, Heckenlively JR, Gordon LK. Frequency of anti-retinal antibodies in normal human serum. J Neuroophthalmol 2008;28:5-11.

47. Kim SJ, Toma HS, Thirkill CE, Dunn JP. Cancer-associated retinopathy with retinal periphlebitis in a patient with ovarian cancer. Ocul Immunol Inflamm 2010;18:107-109.

48. Chin MS, Caruso RC, Detrick B, Hooks JJ. Autoantibodies to p75/LEDGF, a cell survival factor, found in patients with atypical retinal degeneration. J Autoimmun 2006;27:17-27.

\section{Legends}

Figure 1.

Electroretinogram of a patient with paraneoplastic retinopathy. Most patients showed reduced responses on both photopic and scotopic ERGs. Only case 6 showed normal scotopic responses. The numbers are matched to those presented in Table 1. 


\section{Figure 2.}

Color fundus images (left row), fundus autofluorescence images (middle row) and spectral domain-optical coherence tomography (SD-OCT) images (right row) of 8 cases with paraneoplastic retinopathy.

The numbers are matched to those in the Table. All images are from the right eye. SD-OCT images are horizontal scan of case 2, 5, 6, and 8 and vertical scan of case 1, 3, 4, and 7. Autofluorescent images revealed the abnormal hyperand/or hypo- autofluorescent lesions with an inter-individual variability in six cases. The SD-OCT images revealed retinal degeneration in all eyes.

\section{Figure 3.}

Western blot of mouse retina stained with patients' sera as the first antibodies. $10 \%$ SDS-PAGE is shown and the numbers are matched to those in the Table. Whether the bands were specific to retina or not was judged using lysates of other organs (graphic data not shown). The arrowhead shows retina-specific antibodies. The bands over $150 \mathrm{kDa}$ were detected by $8 \%$ SDS-PAGE and they were not shown in this image.

\section{Figure 4.}

Comparison of spectral domain optical coherene tomography (SD-OCT) and immunohistochemistry (IHC) images of patients with paraneoplastic retinopathy. The numbers are matched to those in the Table. The top shows OCT images at higher magnification (1-8). Panels $\mathrm{A}-\mathrm{H}$ are $\mathrm{IHC}$ images that show mouse retina stained with patient serum as the first antibody (green). Cell nuclei are visualized with DAPI (blue). S-100 protein was also used to label the Müller cell in case 1 (red). The control is presented on the bottom left panel. SD-OCT revealed abnormalities in external limiting membrane (ELM) or inner segment/outer segment junction (IS/OS) in all cases. IHC images showed that patients' sera contained antibodies that bind to various retinal proteins. White arrows indicate the site where anti-retinal antibodies in patient's sera reacted to retinal tissue. The scale bar represents $20 \mu \mathrm{m}$. GCL, ganglion cell layer; IPL, inner plexiform layer; INL, inner nuclear layer; OPL, outer plexiform layer; ONL, outer nuclear layer; IS, inner segment of photoreceptor; OS, outer segment of photoreceptor. 


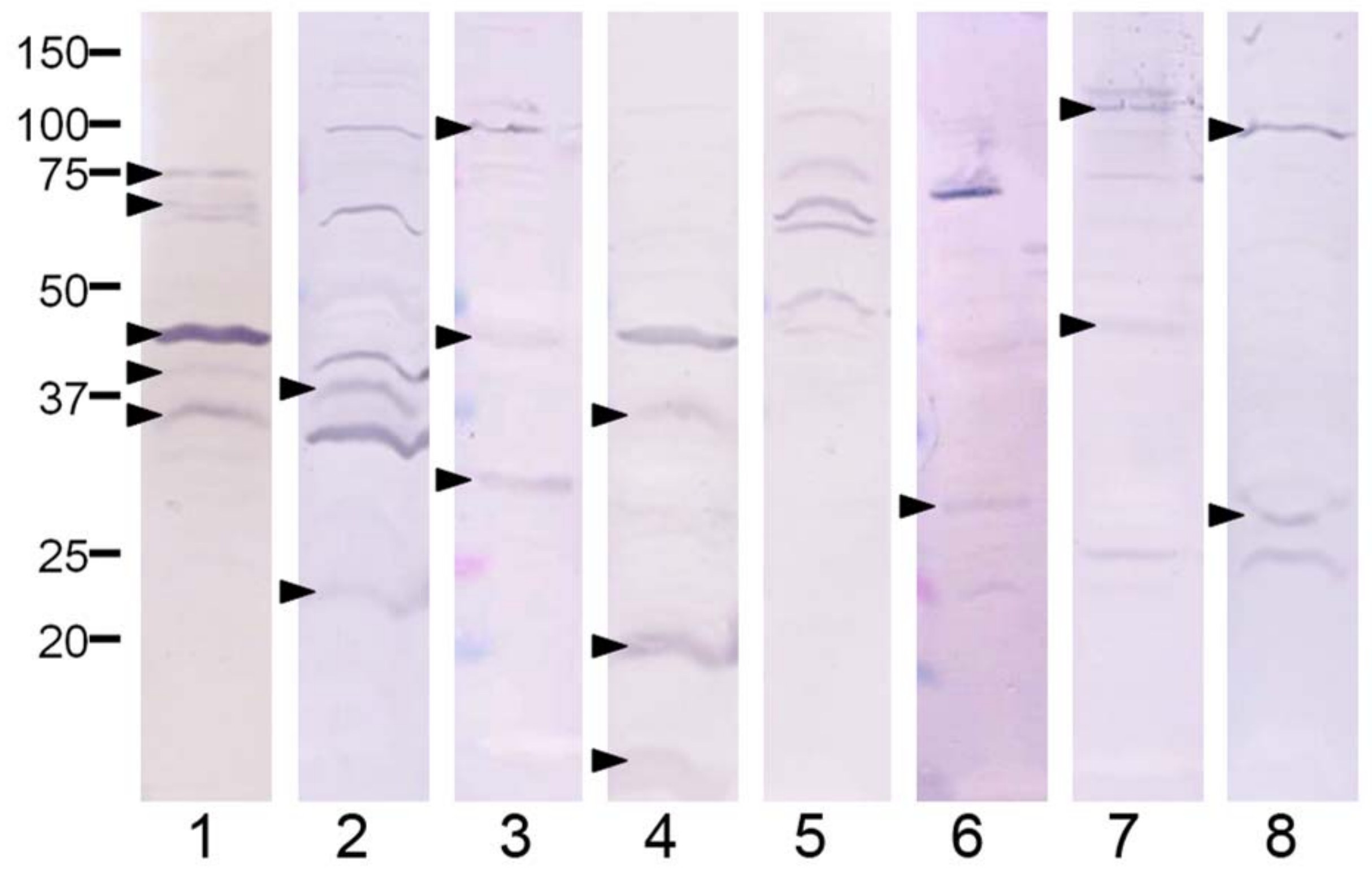

Figure 1 


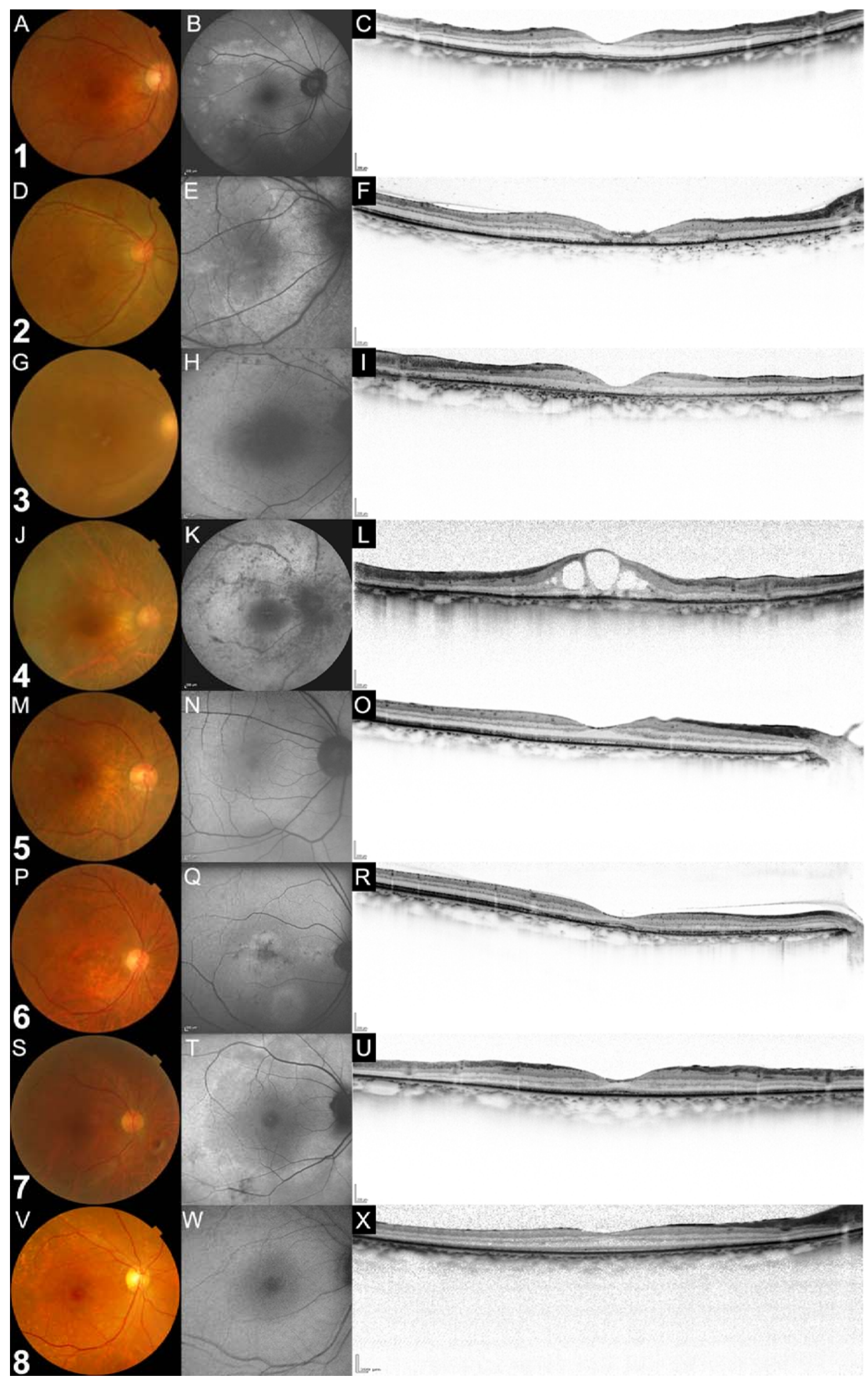

Figure 2 


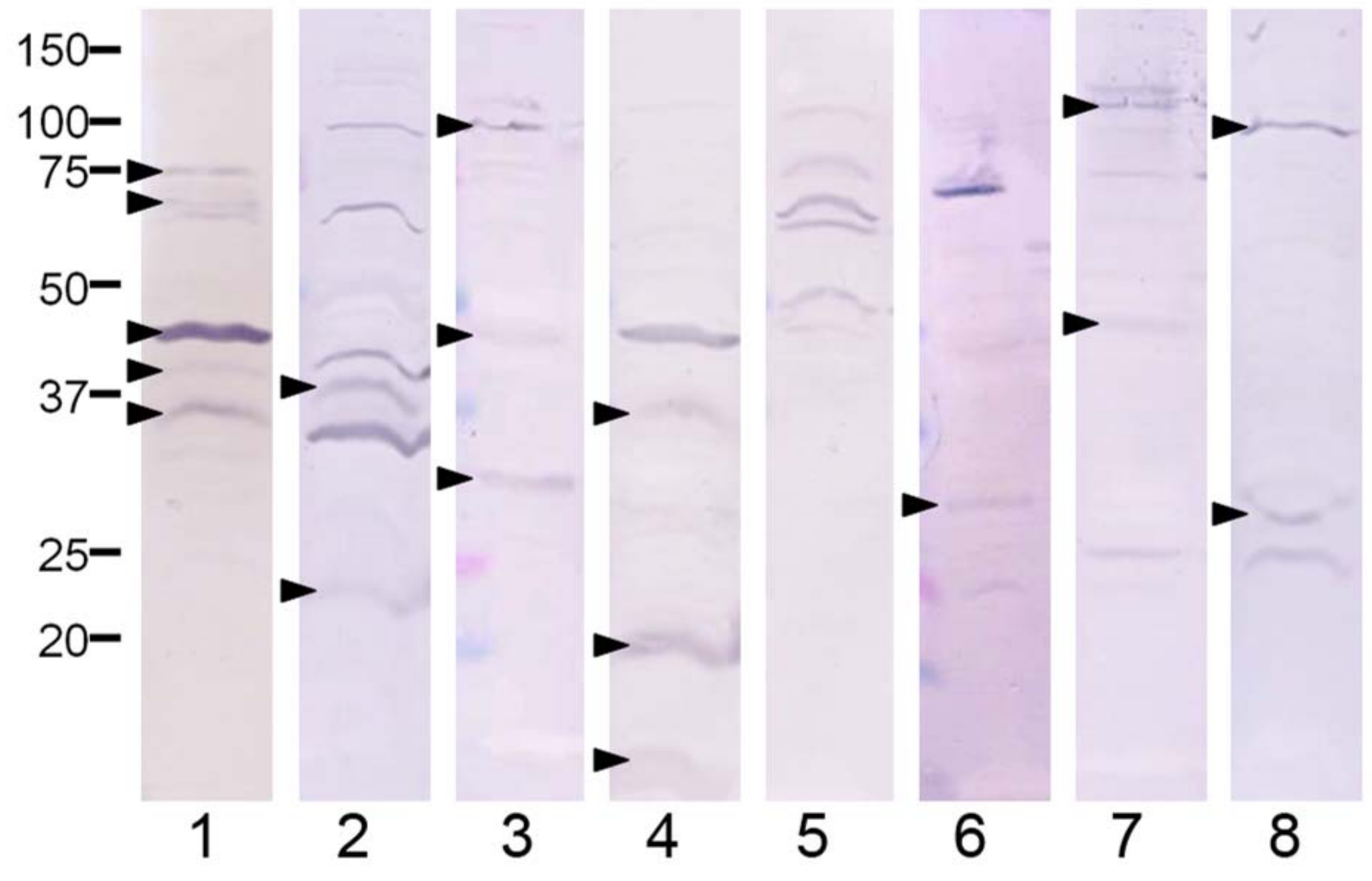

Figure 3 


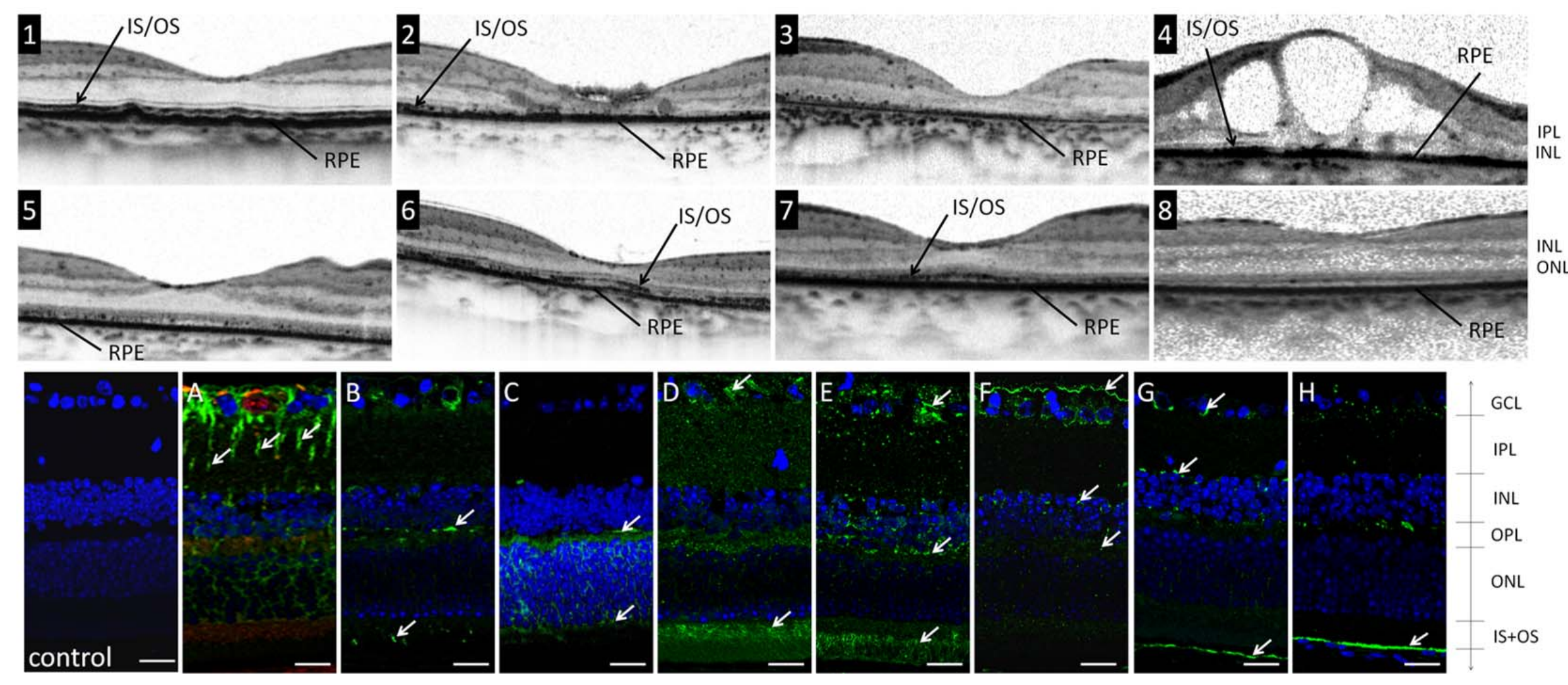

Figure 4 
Table. Clinical Characteristics and Treatment of Neoplasm and Pareneoplastic Retinopathy and Result of Western Blot Analysis of 8 Cases.

\begin{tabular}{|c|c|c|c|c|c|c|c|c|c|c|c|c|}
\hline \multirow{2}{*}{$\begin{array}{l}\text { Case No., } \\
\text { Age at } \\
\text { Diagnosis, } \\
\text { Sex }\end{array}$} & \multirow{2}{*}{$\begin{array}{l}\text { Chief } \\
\text { Complaint }\end{array}$} & \multicolumn{2}{|c|}{ Initial BCVA } & \multirow{2}{*}{ Visual Fields } & \multirow{2}{*}{$\begin{array}{l}\text { Leakage } \\
\text { in } \mathrm{FA}\end{array}$} & \multirow[b]{2}{*}{ ERG } & \multicolumn{2}{|c|}{ Tumor } & \multirow[b]{2}{*}{ Treatment of Neoplasm } & \multirow[b]{2}{*}{ Treatment of Retinopathy } & \multirow{2}{*}{$\begin{array}{c}\text { Serum Examination } \\
\mathrm{WB}(\mathrm{kD})^{*} / \\
\mathrm{IHC}\end{array}$} & \multirow[b]{2}{*}{ Course } \\
\hline & & $\mathrm{R}$ & L & & & & Past History & New Discovery & & & & \\
\hline $\begin{array}{c}\text { Case } 1 \\
85 \mathrm{M}\end{array}$ & Defect of VF & 1.5 & 1.0 & $\begin{array}{l}\text { B) Small islands } \\
\text { scotoma }\end{array}$ & & Subnormal & $\begin{array}{l}\text { Bladder cancer and } \\
\text { Liposarcoma in } \\
\text { mediastinum }\end{array}$ & SCLC & Chemotherapy & $\begin{array}{c}\text { Oral Prednisolone } \\
\text { (Corticosteroid } 25 \mathrm{mg} / \text { day) }\end{array}$ & $\begin{array}{c}36,39,43,64,74,217 / \\
\text { Müller cell }\end{array}$ & Died within 3 months \\
\hline $\begin{array}{c}\text { Case2 } \\
64 \mathrm{~F}\end{array}$ & $\begin{array}{l}\text { Defect of VF and } \\
\text { metamorphopsia }\end{array}$ & 0.4 & 0.1 & $\begin{array}{l}\text { R) Ring scotoma } \\
\text { L) Cental scotoma }\end{array}$ & + & Subnormal & no specific finding & SCLC & $\begin{array}{l}\text { Chemotherapy and } \\
\text { Radiation therapy }\end{array}$ & $\begin{array}{c}\text { Oral Prednisolone } \\
\text { (Corticosteroid } 30 \mathrm{mg} / \text { day) }\end{array}$ & $\begin{array}{c}23,33,37,38,60,97,215 / \\
\text { OS of photoreceptor } \\
\text { (s-cone) }\end{array}$ & $\begin{array}{l}\text { VA stable } \\
\text { for } 2 \text { years }\end{array}$ \\
\hline $\begin{array}{c}\text { Case3 } \\
57 \mathrm{M}\end{array}$ & $\begin{array}{c}\text { Decrease of VA } \\
\text { and } \\
\text { contraction of VF }\end{array}$ & 0.15 & 0.01 & $\begin{array}{l}\text { R) Concentric } \\
\text { contraction } \\
\text { L) Central scotoma }\end{array}$ & + & Subnormal & Thymoma & no specific finding & Surgery & $\begin{array}{l}\text { Steroid therapy (pulse+oral), } \\
\text { Plasmapheresis and IVIg }\end{array}$ & $\begin{array}{c}29,43,100 / \\
O P L \text { and } \\
\text { IS of photoreceptor }\end{array}$ & Died within 1 year \\
\hline $\begin{array}{c}\text { Case4 } \\
81 \mathrm{~F}\end{array}$ & Decrease of VA & 0.09 & $\begin{array}{l}40 \mathrm{~cm} \\
/ \mathrm{HM}\end{array}$ & B) Central scotoma & + & $\begin{array}{l}\text { Subnormal in cone } \\
\text { NR in remainder }\end{array}$ & no specific finding & thymoma & Non-treatment & $\begin{array}{l}\text { Ophthalmic solution } \\
\text { (Betamethasone sodium } \\
\text { phosphate) }\end{array}$ & $\begin{array}{l}17,21,34,41 / \\
\text { GCL and IS of } \\
\text { photoreceptor }\end{array}$ & Drop out \\
\hline $\begin{array}{l}\text { Case5 } \\
78 \mathrm{~F}\end{array}$ & $\begin{array}{l}\text { Decrease of VA } \\
\text { and photopsia }\end{array}$ & 0.15 & 0.08 & $\begin{array}{l}\text { R) Ring scotoma } \\
\text { L) Islands scotoma }\end{array}$ & + & Subnormal & no specific finding & $\begin{array}{l}\text { Pancreatic } \\
\text { neuroendocrine } \\
\text { neoplasm }\end{array}$ & Surgery & Steroid therapy(pulse+oral) & $\begin{array}{c}46,48,60,63,98,189,222 / \\
\text { GCL,OPL } \\
\text { and OS of photoreceptor }\end{array}$ & $\begin{array}{l}\text { VA declined to } 0.03 \text { in } \\
\text { both eyes in } 9 \text { months }\end{array}$ \\
\hline $\begin{array}{c}\text { Case6 } \\
59 \mathrm{M}\end{array}$ & $\begin{array}{c}\text { Decrease of VA } \\
\text { and } \\
\text { night blindness }\end{array}$ & 0.8 & 1.2 & $\begin{array}{l}\text { R) Island scotoma } \\
\text { L) N.P. }\end{array}$ & - & $\begin{array}{l}\text { R) Subnormal } \\
\text { L) Normal }\end{array}$ & Renal cancer & Brain metastasis & $\begin{array}{l}\text { Renal :Operation } \\
\text { Brain: Non-treatment }\end{array}$ & Non-treatment & $\begin{array}{c}34,71,94,102 / \\
\text { GCL, ,INL and OPL }\end{array}$ & $\begin{array}{l}\text { VA stable } \\
\text { for } 2 \text { years }\end{array}$ \\
\hline $\begin{array}{c}\text { Case } 8 \\
68 \mathrm{~F}\end{array}$ & $\begin{array}{l}\text { Decrease of VA } \\
\text { and photopsia }\end{array}$ & 0.3 & 0.03 & $\begin{array}{l}\text { B) Central and ring } \\
\text { scotoma }\end{array}$ & - & Subnormal & no specific finding & Colon cancer & Surgery & Non-treatment & $\begin{array}{l}25,30,90,174 / \\
\text { RPE }\end{array}$ & $\begin{array}{l}\text { VA declined to hand } \\
\text { motion in both eyes } \\
\text { in } 3 \text { years }\end{array}$ \\
\hline
\end{tabular}

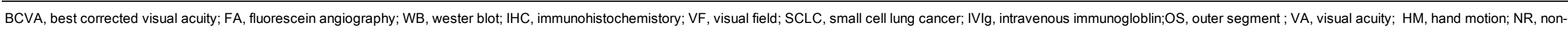
recordable; GCL, ganglion cell layer; IS, innner segment; OPL, outer plexiform layer; NP, not particular; INL, inner nuclear layer; RPE, retinal pigment epithelium. *Specific antibodies against retina or brain are marked in bold. 